\title{
Towards quantifying psychiatric diagnosis using machine learning algorithms and big fMRI data
}

\author{
Fahad Saeed
}

Correspondence:

fahad.saeed@wmich.edu Department of Computer Science, Western Michigan University, Kalamazoo MI 49008, USA

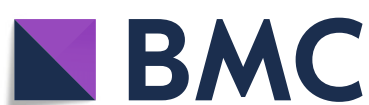

\begin{abstract}
Attention Deficit Hyperactivity Disorder (ADHD) is one of the most common brain disorders among children and is very difficult to diagnose using current methods. Similarly other mental disorders are subject to the same systematic errors with sufficient evidence of diagnostic errors as well as over-prescribing of drugs due to misdiagnosis. For most mental health disorders there is no quantitative method that will inform the presence or absence of a given mental disorder. We argue that definitive and quantitative diagnostic tests are necessary for ADHD and other mental disorders. To this end, big data Functional Magnetic Resonance Imaging (fMRI) and machine learning algorithms can be instrumental in changing the way psychiatric disorders are diagnosed and treated. We briefly discuss our recent research efforts and future directions for a quantitative gold standard tests for psychiatric diagnosis.
\end{abstract}

Keywords: Machine learning and data mining, ADHD, fMRl, Big data, High performance computing

\section{Current and future research directions}

It is widely known that defining and diagnosing mental disorders is a difficult process due to overlapping nature of symptoms, and lack of a biological test that can serve as a definite and quantified gold standard [1]. To this end, Attention Deficit Hyperactivity Disorder (ADHD) is one of the most common brain disorders among children and relies on the identification of abnormal mental characteristics. ADHD is notoriously difficult to diagnose, especially in children, with sufficient evidence of diagnostic errors as well as over-prescribing of drugs due to misdiagnosis [2]. The current psychiatric diagnosis is based purely on behavioural observation (DSM-5/ICD-10) but lacks biological and/or genetic validity and is prone to errors [1]. For most mental health disorders definitive and quantitative diagnostic tests which can detect the presence or the absence of a specific psychiatric disorder(s) are non-existent $[1,3]$.

Functional Magnetic Resonance Imaging (fMRI) is a non-invasive technique for studying the brain functional activities and is based on Blood Oxygen Level Dependent (BOLD) contrast [4]. During the fMRI scan, a series of images are taken using a scanner while the subject to a specific task such as resting, or doing various pre-determined tasks. The

(c) The Author(s). 2018 Open Access This article is distributed under the terms of the Creative Commons Attribution 4.0 International License (http://creativecommons.org/licenses/by/4.0/), which permits unrestricted use, distribution, and reproduction in any medium, provided you give appropriate credit to the original author(s) and the source, provide a link to the Creative Commons license, and indicate if changes were made. The Creative Commons Public Domain Dedication waiver (http:// creativecommons.org/publicdomain/zero/1.0/) applies to the data made available in this article, unless otherwise stated. 
result of brain scanning is a series of low resolution images over time which shows the activity of the brain and can allow us to develop a highly informative brain connectome. There is evidence that ADHD, Bipolar Disorder (BD) and Schizophrenia have characteristics that differ in the regional and global connectivity of the brain when studied under resting state Functional Magnetic Resonance Imaging or fMRI [1, 3, 5].

As data-scientists, along with our colleagues from psychiatry and neuroscience, the overarching question that we have been posing is as follows: Can we diagnose a person with ADHD (or other mental disorders) using fMRI scans using techniques from machine learning and novel algorithmic designs?. If possible, such a test will provide a definitive quantitative 'gold standard' test for diagnosing ADHD and other disorders.

In this endeavor machine-learning, especially deep-learning algorithms, have the potential to show exceptional promise [6-9]. To this end, we have been successful in developing a machine learning algorithm that allow us to classify fMRI ADHD scans from normal healthy brain scans without using any demographic information. Our recently proposed technique is based on computing similarity between two multivariate time series along with k-Nearest-Neighbor classifier. We designed a model selection scheme called J-Eros which is able to pick the optimum value of $\mathrm{k}$ for $\mathrm{k}$-Nearest-Neighbor from the training data. Our results show a 20\% increase in accuracy, with superior sensitivity and specificity, as compared to the state of the art algorithms in classifying ADHD using open-data ADHD-200 that is available to the community [10]. We expect to introduce deep-learning based machine learning algorithms which will help us quantify psychiatric evaluation for ADHD, Bipolar Disorder, and Schizophrenia.

Compute time for analyzing big fMRI data is a bottleneck in introducing such techniques in a clinical setting $[11,12]$. High performance computing (HPC) techniques will help ease the computational load in big fMRI data analysis for clinical and diagnostic purposes [13]. Among parallel computing techniques, Graphic Processing Unit (GPU) architecture provides a high degree of parallelism while being less expensive. Therefore, the focus of our research has been towards designing GPU based parallel computing algorithms for analyzing big fMRI data and considering pairwise relations between all voxels (the smallest cubic unit in fMRI data) without spatial or temporal constraint. In this way, voxels from different regions of brain which have similar spatiotemporal behaviour could be placed in the same cluster. The resulting clusters are regions which are functionally homogeneous and would give a more system-wide view of brain connectivity. This will allow neuroscientists to study the regional and global connectivity of the brain in much fine-grained detail than is currently possible. To this end, we have recently presented a GPU based strategy that allows us to calculate the functional connectivity using Pearson correlations for big fMRI data [4].

Such quantified psychiatric evaluations based on data science, big data, and algorithms can increase the accuracy of diagnosis, prognosis, and treatments of difficult to assess mental disorders. It will be an immense service for mankind if we are successful in developing a quantitative framework for big fMRI data to identify brain disorders from healthy brains. These algorithms will play a vital role in peering into mental disorders and will be akin to the role that microscope played for diagnostic medicine in the early 19 th century. 
Author's contributions

The author read and approved the final manuscript.

Competing interests

The author declares that he has no competing interests.

\section{Publisher's Note}

Springer Nature remains neutral with regard to jurisdictional claims in published maps and institutional affiliations.

Received: 9 May 2018 Accepted: 16 May 2018

Published online: 31 May 2018

\section{References}

1. N. C. C. for Mental Health UK, et al. Attention deficit hyperactivity disorder: diagnosis and management of adhd in children, young people and adults. Br Psychol Soc. 2018. https://www.ncbi.nlm.nih.gov/pubmed/29634174.

2. Dunlop AJ, Newman LK. Adhd and psychostimulants—overdiagnosis and overprescription. Med J Aust. 2016:204(4):139.

3. N. C. C. for Mental Health UK, et al. Bipolar disorder: The nice guideline on the assessment and management of bipolar disorder in adults, children and young people in primary and secondary care. Br Psychol Soc. 2018. https:// www.ncbi.nlm.nih.gov/books/NBK498655/.

4. Eslami T, Saeed F. Fast-GPU-PCC: A GPU-based technique to compute pairwise pearson's correlation coefficients for time series data—fMRI study. High-Throughput. 2018;7(2). https://doi.org/10.3390/ht7020011. http://www.mdpi. com/2571-5135/7/2/11.

5. Argyelan M, Ikuta T, DeRosse P, Braga RJ, Burdick KE, John M, Kingsley PB, Malhotra AK, PR Szeszko. Resting-state fmri connectivity impairment in schizophrenia and bipolar disorder. Schizophr Bull. 2013;40(1):100-10.

6. Mahmud M, Kaiser MS, Hussain A, Vassanelli S. Applications of deep learning and reinforcement learning to biological data. IEEE Trans Neural Netw Learn Syst. 2018;29(6):2063-2079. https://doi.org/10.1109/TNNLS.2018. 2790388.

7. Shi B, Chen Y, Zhang P, Smith CD, Liu J, Initiative ADN, et al. Nonlinear feature transformation and deep fusion for alzheimer's disease staging analysis. Pattern Recognit. 2017;63:487-98.

8. Suk H-I, Lee S-W, Shen D, Initiative ADN, et al. Hierarchical feature representation and multimodal fusion with deep learning for ad/mci diagnosis. Neurolmage. 2014;101:569-82.

9. Sarraf S, Tofighi G, et al. DeepAD: Alzheimer's disease classification via deep convolutional neural networks using MRI and fMRI. bioRxiv. 2016. Cold Spring Harbor Laboratory. https://doi.org/10.1101/070441. https://www.biorxiv. org/content/early/2016/08/21/070441.full.pdf.

10. Eslami T, Saeed F. Similarity based classification of adhd using singular value decomposition. In: Proceedings of ACM International Conference on Computing Frontiers. NY: Association of Computing Machinery (ACM); 2018

11. Chen S, Huang L, Qiu H, Nebel MB, Mostofsky SH, Pekar JJ, Lindquist MA, Eloyan A, Caffo BS. Parallel group independent component analysis for massive fmri data sets. PloS ONE. 2017;12(3):e0173496.

12. Stollenga MF, Byeon W, Liwicki M, Schmidhuber J. Parallel multi-dimensional LSTM, with application to fast biomedical volumetric image segmentation. In: Proceedings of the 28th International Conference on Neural Information Processing Systems - Volume 2. Cambridge: MIT Press; 2015. p. 2998-3006. http://dl.acm.org/citation. cfm?id=2969442.2969574.

13. Phinyomark A, Ibáñez-Marcelo E, Petri G. Resting-state fmri functional connectivity: Big data preprocessing pipelines and topological data analysis. IEEE Trans Big Data. 2017;3(4):415-28.

Ready to submit your research? Choose BMC and benefit from:
- fast, convenient online submission
- thorough peer review by experienced researchers in your field
- rapid publication on acceptance
- support for research data, including large and complex data types
- gold Open Access which fosters wider collaboration and increased citations
- maximum visibility for your research: over 100M website views per year
At BMC, research is always in progress.
Learn more biomedcentral.com/submissions

